\title{
Tarifbindung und betriebliche Interessen- vertretung: Aktuelle Ergebnisse aus dem IAB-Betriebspanel 2007
}

\author{
Peter Ellguth \\ Susanne Kohaut
}

Der Flächentarifvertrag wird in Deutschland auch aufgrund der Mindestlohndebatte wieder stark diskutiert. Der Anteil der Betriebe, die an einen solchen Tarifvertrag gebunden sind, ist seit Jahren rückläufig, wie hier bereits mehrfach berichtet. ${ }^{1}$ Bis in die 1990er Jahre wurden Löhne und Arbeitsbedingungen in Westdeutschland überwiegend branchenweit geregelt. Die dominierende Rolle des branchenweiten Verbandstarifvertrages und der ihn schlieBenden Tarifparteien hat seither abgenommen, dagegen haben betriebsbezogene Regelungen und die betriebliche Interessenvertretung an Bedeutung gewonnen. Heute ist die Situation in West- wie Ostdeutschland durch eine große Differenzierung und Dezentralisierung gekennzeichnet.

\begin{abstract}
An dieser Stelle wollen wir die „Tradition" unserer Berichterstattung in den WSI-Mitteilungen fortsetzen und aktuelle Ergebnisse zur Tarifbindung und der betrieblichen Interessenvertretung präsentieren. Die einzige repräsentative Datenquelle, die entsprechende Daten für die Gesamtwirtschaft liefert, ist das IAB-Betriebspanel. Es enthält für Westdeutschland Informationen zur Existenz eines Betriebsrats seit 1993, nach der Tarifbindung der Betriebe wird ab 1995 gefragt. 1996 wurden auch ostdeutsche Betriebe mit in die Erhebung einbezogen. Die Ergebnisse für 2007 beruhen auf den Angaben von rund 15.500 west- und ostdeutschen Betrieben. Aufgrund des Aufbaus der Zufallsstichprobe sind die Ergebnisse repräsentativ für die rund 2 Mio. Betriebe mit mindestens ei-
\end{abstract}

\begin{tabular}{|c|c|c|c|c|c|c|}
\hline \multirow[t]{2}{*}{ Branche } & \multicolumn{2}{|c|}{$\begin{array}{l}\text { Branchen- } \\
\text { tarifvertrag }\end{array}$} & \multicolumn{2}{|c|}{$\begin{array}{c}\text { Firmen- } \\
\text { tarifvertrag }\end{array}$} & \multicolumn{2}{|c|}{$\begin{array}{c}\text { Kein Tarifvertrag } \\
\text { (davon Orientierung } \\
\text { an einem Tarifvertrag) }\end{array}$} \\
\hline & West & Ost & West & Ost & West & Ost \\
\hline Landwirtschaft u.a. & 56 & 15 & * & * & $43(40)$ & $83(49)$ \\
\hline Bergbau/Energie & 81 & 57 & 10 & 32 & $9(56)$ & $11(66)$ \\
\hline $\begin{array}{l}\text { Grundstoffverarbeitung } \\
\text { (Produktionsgüter) }\end{array}$ & 64 & 32 & 11 & 12 & $25(65)$ & $56(47)$ \\
\hline Investitionsgüter & 60 & 25 & 8 & 17 & $32(67)$ & $59(57)$ \\
\hline Verbrauchsgüter & 51 & 29 & 10 & 17 & $40(56)$ & $54(49)$ \\
\hline Baugewerbe & 75 & 48 & 2 & 8 & $23(64)$ & $44(63)$ \\
\hline Handel/Reparatur & 48 & 24 & 5 & 6 & $47(57)$ & $69(50)$ \\
\hline Verkehr/Nachrichten & 45 & 33 & 11 & 17 & $44(46)$ & $50(44)$ \\
\hline Kredit/Versicherung & 86 & 73 & 4 & * & $10(63)$ & $26(52)$ \\
\hline Dienste für Unternehmen & 37 & 45 & 6 & 7 & $57(37)$ & $48(32)$ \\
\hline sonstige Dienste & 53 & 36 & 8 & 17 & $39(53)$ & $48(53)$ \\
\hline Organisation ohne Erwerbszweck & 49 & 32 & 12 & 15 & $39(68)$ & $54(55)$ \\
\hline Öffentliche Verwaltung & 89 & 79 & 8 & 18 & $4(91)$ & $3(42)$ \\
\hline Beschäftigte gesamt & 56 & 41 & 7 & 13 & $37(53)$ & $47(49)$ \\
\hline
\end{tabular}

(c) WSI Mitteilungen 2002-2008

Diese Datei und ihr Inhalt sind urheberrechtlich geschützt. Nachdruck und Verwertung (gewerbliche Vervielfältigung, Aufnahme in elektronische Datenbanken, Veröffentlichung online oder offline) sind nicht gestattet. nem sozialversicherungspflichtig Beschäftigten. Insgesamt sind in diesen Betrieben 33,6 Mio. Personen beschäftigt.

\section{AUSMAß UND ENTWICKLUNG DER TARIFBINDUNG}

Überbetriebliche Verbands- oder Flächentarifverträge, die zwischen Arbeitgeberverbänden und Gewerkschaften branchenweit ausgehandelt werden, spielen in Deutschland immer noch eine wichtige Rolle bei der Regelung von Löhnen und Arbeitsbeziehungen. Zwar können diese auch auf Betriebs- oder Unternehmensebene (Firmen-

\footnotetext{
Vgl. zuletzt Ellguth, P./Kohaut, S. (2005): Tarifbindung und betriebliche Interessenvertretung - Aktuelle Ergebnisse aus dem IAB-Betriebspanel, in: WSI-Mitteilungen 7, S. 398-402: Ellguth, P./Kohaut, S. (2007): Tarifbindung und betriebliche Interessenvertretung - Aktuelle Ergebnisse aus dem IAB-Betriebspanel 2006, in: WSI-Mitteilungen 9. S. 511-514.
}

Peter Ellguth ist wissenschaftlicher Mitarbeiter im Institut für Arbeitsmarkt- und Berufsforschung (IAB) der Bundesagentur für Arbeit in der Projektgruppe IAB-Betriebspanel. Arbeitsschwerpunkte: Arbeitsbeziehungen und betriebliche Arbeitszeitpolitik. e-Mail: peter.ellguth@iab.de Susanne Kohaut, Dr., ist wissenschaftliche Mitarbeiterin im Institut für Arbeitsmarktund Berufsforschung (IAB) der Bundesagentur für Arbeit in der Projektgruppe IAB-Betriebspanel.

Arbeitsschwerpunkte: Tarifbindung, Neugründen und betriebliche Innovationen. e-Mail: susanne.kohaut@iab.de 
tarifverträge) oder in individuellen Arbeitsverträgen geregelt werden, im deutschen Arbeitsrecht haben tarifvertragliche Regelungen jedoch Vorrang gegenüber Betriebsvereinbarungen und Einzelarbeitsverträgen. So können Regelungen in Tarifverträgen auch als Mindestarbeitsbedingungen interpretiert werden.

Im Folgenden werden aktuelle Ergebnisse des IAB-Betriebspanels für das Jahr 2007 dargestellt, wobei zwischen der Tarifbindung der Betriebe und der Beschäftigten unterschieden wird. Betrachtet man zunächst die Beschäftigten, so haben im Jahr 2007 hochgerechnet rund $56 \%$ der westdeutschen und etwa $41 \%$ der ostdeutschen Beschäftigten in einem Betrieb gearbeitet, der einem Branchentarifvertrag unterlag (Tabelle 1). Firmentarifverträge galten für $7 \%$ der westdeutschen und $13 \%$ der ostdeutschen Beschäftigten. Für gut ein Drittel der westdeutschen und $47 \%$ der ostdeutschen Arbeitnehmer gab es keinen Tarifvertrag. Jeweils rund die Hälfte dieser Arbeitnehmer wurde jedoch indirekt von Tarifverträgen erfasst, da sich ihre Betriebe nach eigenen Angaben daran orientierten. Das Ausmaß der Tarifbindung variiert zwischen den Wirtschaftszweigen stark, wobei sich die Bedeutung für die einzelnen Branchen von Jahr zu Jahr wenig ändert.

Aufgrund der starken Größenabhängigkeit der Tarifbindung ergibt sich auf Ebene der Betriebe, dass hochgerechnet rund $36 \%$ der westdeutschen, aber nur $20 \%$ der ostdeutschen Betriebe durch Branchentarifverträge gebunden waren. Haus- oder Firmentarifverträge galten für $3 \%$ der Betriebe in den alten und etwa $4 \%$ der Betriebe in den neuen Bundesländern (Tabelle 2). Der Rest, also etwa $62 \%$ der westdeutschen und drei Viertel der ostdeutschen Betriebe, war nicht tarifgebunden. Allerdings gaben etwa $40 \%$ der nicht tarifgebundenen Betriebe in beiden Landesteilen an, sich in ihren Einzelarbeitsverträgen an bestehenden Branchentarifen zu orientieren.

Betrachtet man die Tarifbindung der Betriebe nach ihrer Größe (Tabelle 3), so zeigt sich wie in den Vorjahren, dass der Deckungsgrad mit zunehmender Größe steigt. Das gilt sowohl für die alten als auch für die neuen Bundesländer, wenn auch auf unterschiedlichem Niveau. Während für die Kleinbetriebe die Branchentarifverträge eine untergeordnete Rolle spielen, sind Großbetriebe mit über 500 Beschäftigten in der Mehrheit tarifgebunden. Ebenso

Tabelle 2: Tarifbindung der Betriebe in West- und Ostdeutschland 2007 - Anteil der jeweils betroffenen Betriebe in \% -

\begin{tabular}{|c|c|c|c|c|c|c|}
\hline \multirow[t]{2}{*}{ Branche } & \multicolumn{2}{|c|}{$\begin{array}{l}\text { Branchen- } \\
\text { tarifvertrag }\end{array}$} & \multicolumn{2}{|c|}{$\begin{array}{l}\text { Firmen- } \\
\text { tarifvertrag }\end{array}$} & \multicolumn{2}{|c|}{$\begin{array}{c}\text { Kein Tarifvertrag } \\
\text { (davon Orientierung } \\
\text { an einem Tarifvertrag) }\end{array}$} \\
\hline & West & Ost & West & Ost & West & Ost \\
\hline Landwirtschaft u.a. & 40 & 8 & * & $*$ & $60(37)$ & $92(38)$ \\
\hline Bergbau/Energie & 55 & 38 & 4 & 15 & $42(56)$ & $47(67)$ \\
\hline $\begin{array}{l}\text { Grundstoffverarbeitung } \\
\text { (Produktionsgüter) }\end{array}$ & 42 & 18 & 4 & 5 & $54(51)$ & $77(40)$ \\
\hline Investitionsgüter & 30 & 17 & 3 & 6 & $67(55)$ & $78(49)$ \\
\hline Verbrauchsgüter & 46 & 24 & 3 & 3 & $51(46)$ & $73(47)$ \\
\hline Baugewerbe & 61 & 38 & 1 & 4 & $38(56)$ & $58(59)$ \\
\hline Handel/Reparatur & 34 & 15 & 2 & 4 & $63(44)$ & $81(40)$ \\
\hline Verkehr/Nachrichten & 34 & 15 & 4 & 6 & $62(46)$ & $80(42)$ \\
\hline Kredit/Versicherung & 42 & 32 & 4 & * & $54(45)$ & $66(27)$ \\
\hline Dienste für Unternehmen & 13 & 14 & 1 & 4 & $85(29)$ & $83(28)$ \\
\hline sonstige Dienste & 36 & 16 & 3 & 5 & $61(43)$ & $79(44)$ \\
\hline Organisation ohne Erwerbszweck & 40 & 20 & 8 & 9 & $52(42)$ & $72(33)$ \\
\hline Öffentliche Verwaltung & 85 & 85 & 7 & 5 & $8(79)$ & $11(51)$ \\
\hline Betriebe gesamt & 36 & 20 & 3 & 4 & $62(42)$ & $76(41)$ \\
\hline
\end{tabular}

Tabelle 3: Tarifbindung der Betriebe in West- und Ostdeutschland 2007 nach Betriebsgröße - Anteil der jeweils betroffenen Betriebe in \% -

\begin{tabular}{|c|c|c|c|c|c|c|}
\hline \multirow[t]{2}{*}{ Betriebe } & \multicolumn{2}{|c|}{ Branchentarifvertrag } & \multicolumn{2}{|c|}{ Firmentarifvertrag } & \multicolumn{2}{|c|}{$\begin{array}{c}\text { Kein Tarifvertrag } \\
\text { (davon Orientierung } \\
\text { an einem Tarifvertrag) }\end{array}$} \\
\hline & West & Ost & West & Ost & West & Ost \\
\hline 1 bis 9 & 30 & 16 & 2 & 3 & $68(37)$ & 81 (39) \\
\hline 10 bis 49 & 46 & 28 & 4 & 7 & $50(55)$ & $65(48)$ \\
\hline 50 bis 199 & 54 & 44 & 8 & 14 & $39(60)$ & $43(54)$ \\
\hline 200 bis 499 & 68 & 54 & 12 & 20 & $21(66)$ & $26(45)$ \\
\hline 500 und mehr & 77 & 68 & 10 & 22 & $12(67)$ & $10(49)$ \\
\hline Betriebe gesamt & 36 & 20 & 3 & 4 & $62(42)$ & $76(41)$ \\
\hline
\end{tabular}

nimmt die Bedeutung der Haus- bzw. Firmentarifverträge mit steigender Betriebsgröße zu.

Im IAB-Betriebspanel werden die Daten zur Tarifbindung seit 1996 für beide Landesteile erhoben. Die Flächentarifbindung zeigt in den alten wie in den neuen Bundesländern seither rückläufige Tendenz. In Westdeutschland ging die Flächentarifbindung bezogen auf die Beschäftigten von 1996 bis 2007 um 13 Prozentpunkte zurück. In Ostdeutschland belief sich dieser Rückgang sogar auf 15 Prozentpunkte. Bezogen auf die Betriebe betrugen die entsprechenden Rückgänge der Flächentarifbindung im Westen etwa 12 und im Osten rund 9 Prozentpunkte. Wie es sich bereits in den Vorjahren abzeichnete, scheint sich nun die Tarifbindung in Ostdeutschland auf einem niedrigeren Niveau zu stabilisieren, während in Westdeutschland immer noch ein leichter Rückgang zu verzeichnen ist.

Fasst man die beiden Landesteile zusammen, so gilt derzeit in Deutschland für etwa $32 \%$ der Betriebe mit rund $53 \%$ der Beschäftigten direkt ein Branchentarifvertrag. Rund ein Viertel aller Betriebe orientiert sich darüber hinaus an einem Branchentarifvertrag, sodass weitere $20 \%$ der Beschäftigten zumindest indirekt von den Regelungen eines Tarifvertrages erfasst werden. Für einen großen Anteil der Beschäftigten hat damit der Flächentarifvertrag seine Bedeutung ungebrochen behalten.

\section{AKTUELLE ERGEBNISSE ZUR BETRIEB- LICHEN INTERESSENVERTRETUNG}

Wie schon erwähnt, ist das bundesdeutsche duale System der Interessenvertretung durch eine andauernde Verlagerung von Regelungskompetenzen von der überbetrieblichen auf die betriebliche Ebene gekennzeichnet. In der interessierten Öffentlichkeit wird diese Bedeutungsverschiebung in erster Linie unter dem Aspekt der Flexibilisierung des Tarifsystems diskutiert. 


\begin{tabular}{|c|c|c|c|c|c|c|}
\hline \multirow[b]{2}{*}{ Anteile in \% } & \multicolumn{5}{|c|}{ Betriebsgrößenklassen } & \multirow[b]{2}{*}{$\begin{array}{c}\text { Insgesamt } \\
\text { (ab 5 } \\
\text { Besch.) }\end{array}$} \\
\hline & $\begin{array}{c}5 \text { bis } \\
50 \\
\text { Besch. }\end{array}$ & $\begin{array}{c}51 \mathrm{bis} \\
100 \\
\text { Besch. }\end{array}$ & $\begin{array}{c}101 \text { bis } \\
199 \\
\text { Besch. }\end{array}$ & $\begin{array}{c}200 \text { bis } \\
500 \\
\text { Besch. }\end{array}$ & $\begin{array}{c}501 \\
\text { u.m. } \\
\text { Besch. }\end{array}$ & \\
\hline \multicolumn{7}{|c|}{ Westdeutschland (ohne Berlin) } \\
\hline Betriebe mit BR & 6 & 39 & 64 & 79 & 90 & 10 \\
\hline Betriebe mit anderer MA-Vertretung & 9 & 14 & 11 & 11 & 10 & 9 \\
\hline Beschäftigte mit BR & 10 & 40 & 65 & 81 & 92 & 46 \\
\hline Beschäftigte mit andere MA-Vertretung & 11 & 14 & 11 & 11 & 11 & 11 \\
\hline \multicolumn{7}{|c|}{ Ostdeutschland (mit Berlin) } \\
\hline Betriebe mit BR & 6 & 38 & 60 & 74 & 85 & 10 \\
\hline Betriebe mit anderer MA-Vertretung & 4 & 7 & 6 & 12 & 13 & 4 \\
\hline Beschäftigte mit BR & 12 & 39 & 61 & 74 & 88 & 39 \\
\hline Beschäftigte mit anderer MA-Vertretung & 5 & 7 & 6 & 11 & 11 & 7 \\
\hline
\end{tabular}

Die in diesem Zusammenhang nach wie vor geforderte Erweiterung betrieblicher Handlungsspielräume tarifgebundener Betriebe setzt (mehr oder weniger stillschweigend) die Existenz eines kompetenten betrieblichen Verhandlungspartners voraus, der die Interessen der Arbeitnehmer bündelt und vertritt. Aber auch - oder viel mehr gerade auch - für die Betriebe ohne Tarifbindung stellt sich die Frage nach einer (gesetzlich legitimierten) Vertretung der Beschäftigten auf betrieblicher Ebene.

Vor diesem Hintergrund soll zunächst analog zur Tarifbindung ein Eindruck der quantitativen Reichweite der betrieblichen Mitbestimmung, d.h. der Verbreitung von Betriebsräten vermittelt werden. In Anbetracht des zunehmenden Interesses an den Arbeitsbeziehungen in betriebsratslosen Betrieben werden ergänzend auch Zahlen zu betriebsspezifischen Formen der Mitarbeitervertretung, wie sie seit 2003 im IABBetriebspanel erhoben werden, dargestellt. Der Frage, inwieweit diese Einrichtungen ein funktionales Äquivalent zum Betriebsrat sein können, kann an dieser Stelle allerdings nicht nachgegangen werden. Ab- schließend sollen dann beide Ebenen der Tarif- und Mitbestimmungslandschaft gemeinsam betrachtet werden.

In Tabelle 4 sind die aktuellen Daten zur Existenz von Betriebsräten und alternativen Vertretungsformen in der Privatwirtschaft (Betriebe ab fünf Beschäftigte) für West- und Ostdeutschland dargestellt. Für den Anteil der Betriebe mit gesetzlich legitimierter Interessenvertretung wird mit ca. $10 \%$ in beiden Landesteilen 2007 das Niveau des Vorjahres erreicht und das langjährige Niveau bestätigt.

Bei der letztjährigen Berichterstattung hatten wir für Westdeutschland in der Gesamtschau der vergangenen zwölf Jahre einen leichten Abwärtstrend im auf Beschäftigte bezogenen Deckungsgrad konstatiert. Dieser Rückgang setzt sich am aktuellen Rand nicht fort, es wird vielmehr mit $46 \%$ exakt der Vorjahreswert erreicht. In Ostdeutschland bleibt die Reichweite der betrieblichen Mitbestimmung auch 2007 unverändert, wenngleich auf deutlich niedrigerem Niveau (39\%). Die Rede von der weitgehenden Stabilität der quantitativen Basis der Mitbestimmung auf betrieblicher
Ebene findet damit in den aktuellen Zahlen wieder ihre Bestätigung.

Die hinlänglich bekannten größenspezifischen Unterschiede in der Verbreitung von Betriebsräten stellen sich ebenfalls wieder ein. Nur knapp jeder 14. Kleinbetrieb (bis 50 Beschäftigte) verfügt über einen Betriebsrat. Unter den Großbetrieben (über 500 Beschäftigte) ist die Existenz eines Betriebsrats dagegen die Regel und erreicht Anteilswerte von $90 \%$ in Westdeutschland und $85 \%$ in Ostdeutschland. Prägt in betrieblicher Perspektive die große Zahl der Kleinbetriebe das Gesamtbild, so relativiert sich deren Gewicht natürlich bei Betrachtung des auf Beschäftigte bezogenen Deckungsgrads. Die geringere Verbreitung von Betriebsräten im mittel- und großbetrieblichen Segment Ostdeutschlands manifestiert sich in der dort rund 7 Prozentpunkte niedrigeren Reichweite der betrieblichen Mitbestimmung.

Ebenso bestätigen sich die erwarteten starken Unterschiede zwischen einzelnen Branchen und das sowohl in Betriebs- als auch Beschäftigtenperspektive (Tabelle 5). Nach wie vor besteht die größte quantitative Reichweite im traditionell stark mitbestimmten Bereich Bergbau/Energie- und Wasserversorgung und im Kredit- und Versicherungsgewerbe. Das untere Ende bildeten wie gewohnt die vorwiegend kleinbetrieblich strukturierten Branchen des Dienstleitungsbereichs, des Handels und der Bauwirtschaft.

\section{BETRIEBSSPEZIFISCHE FORMEN DER MITARBEITERVERTRETUNG}

Alternativ zu oder auch neben einem Betriebsrat kann es in den Betrieben andere betriebsspezifische Formen der Mitarbeitervertretung geben. Die Verbreitung solcher Einrichtungen, seien es "Runde Tische" oder "Belegschaftssprecher" etc.,

\begin{tabular}{|c|c|c|c|c|c|c|c|c|c|c|c|}
\hline \multirow[b]{2}{*}{ Anteil in \% } & \multirow[b]{2}{*}{$\begin{array}{c}\text { Bergbau/ } \\
\text { Energie } \\
\text { Wasservers. }\end{array}$} & \multirow[b]{2}{*}{$\begin{array}{l}\text { Ver- } \\
\text { brauchs- } \\
\text { güter }\end{array}$} & \multirow[b]{2}{*}{$\begin{array}{l}\text { Produk- } \\
\text { tions- } \\
\text { güter }\end{array}$} & \multirow[b]{2}{*}{$\begin{array}{l}\text { Inves- } \\
\text { titions- } \\
\text { güter }\end{array}$} & \multicolumn{3}{|c|}{ Wirtschaftszweige } & \multirow[b]{2}{*}{$\begin{array}{l}\text { Kredit/ } \\
\text { Ver- } \\
\text { sicherung }\end{array}$} & \multirow[b]{2}{*}{$\begin{array}{l}\text { Unternehmens- } \\
\text { bezogene } \\
\text { Dienstleist. }\end{array}$} & \multirow[b]{2}{*}{$\begin{array}{l}\text { Sonst. } \\
\text { Dienst- } \\
\text { leistungen }\end{array}$} & \multirow[b]{2}{*}{$\begin{array}{c}\text { Insgesamt } \\
\text { (ab 5 } \\
\text { Besch.) }\end{array}$} \\
\hline & & & & & $\begin{array}{l}\text { Bau- } \\
\text { gewerbe }\end{array}$ & Handel & $\begin{array}{l}\text { Verkehr/ } \\
\text { Nach- } \\
\text { richten }\end{array}$ & & & & \\
\hline \multicolumn{12}{|c|}{ Gesamtdeutschland } \\
\hline Betriebe mit $B R$ & 46 & 14 & 22 & 14 & 3 & 10 & 18 & 41 & 9 & 7 & 10 \\
\hline $\begin{array}{l}\text { Betriebe mit anderer } \\
\text { MA-Vertretung }\end{array}$ & 12 & 5 & 6 & 9 & 6 & 8 & 8 & 3 & 8 & 10 & 8 \\
\hline Beschäftigte mit BR & 89 & 50 & 74 & 70 & 18 & 31 & 46 & 80 & 32 & 35 & 45 \\
\hline $\begin{array}{l}\text { Beschäftigte mit anderer } \\
\text { MA-Vertretung }\end{array}$ & 9 & 8 & 11 & 7 & 9 & 10 & 8 & 7 & 9 & 17 & 10 \\
\hline
\end{tabular}


Tabelle 6: Tarifbindung und Betriebsrat 1996-2007*

\begin{tabular}{|c|c|c|c|c|c|c|c|c|c|c|c|c|c|c|}
\hline & \multicolumn{14}{|c|}{ Anteil der jeweils betroffenen Beschäftigten - in \% - } \\
\hline & \multicolumn{7}{|c|}{ Westdeutschland (ohne Berlin) } & \multicolumn{7}{|c|}{ Ostdeutschland (mit Berlin) } \\
\hline & 1996 & 1998 & 2000 & 2002 & 2004 & 2006 & 2007 & 1996 & 1998 & 2000 & 2002 & 2004 & 2006 & 2007 \\
\hline BR und Branchentarif & 41 & 39 & 37 & 37 & 35 & 32 & 32 & 29 & 25 & 25 & 23 & 22 & 19 & 20 \\
\hline BR und Haustarif & $9 * *$ & 6 & 6 & 6 & 6 & 7 & 6 & $13^{* *}$ & 9 & 8 & 10 & 9 & 10 & 10 \\
\hline BR und kein Tarif & & 4 & 7 & 6 & 6 & 7 & 8 & & 5 & 8 & 9 & 9 & 10 & 9 \\
\hline Branchentarif und kein BR & 27 & 28 & 25 & 24 & 24 & 23 & 22 & 22 & 21 & 16 & 15 & 16 & 16 & 16 \\
\hline Haustarif und kein BR & $23^{* *}$ & 2 & 1 & 1 & 1 & 1 & 2 & $36 * *$ & 5 & 3 & 4 & 4 & 4 & 3 \\
\hline Kein Tarif und kein BR & & 21 & 24 & 25 & 27 & 30 & 31 & & 35 & 39 & 40 & 41 & 41 & 42 \\
\hline Gesamt & 100 & 100 & 100 & 100 & 100 & 100 & 100 & 100 & 100 & 100 & 100 & 100 & 100 & 100 \\
\hline
\end{tabular}

zeigt allerdings nicht annähernd die gleiche Stabilität, wie dies die Institution des Betriebsrats auszeichnet. Zwischen den einzelnen Erhebungswellen gibt es z. T. deutliche Schwankungen. Von einem generellen Aufwärtstrend kann nicht die Rede sein. 2007 besaßen $9 \%$ der Betriebe in Westund $4 \%$ in Ostdeutschland eine solche Einrichtung (Tabelle 4). Diese Zahlen liegen über denen von 2003 , aber unter denen von 2005. Wie sich darüber hinaus zeigt, existiert - anders als beim Betriebsrat - eine große Fluktuation unter diesen Gremien, d.h. ein Großteil davon existiert nur über einen relativ kurzen Zeitraum, dafür werden auch viele Jahr für Jahr neu gegründet.

Diese präsentierten Zahlen beziehen sich ebenfalls auf die privatwirtschaftlichen Betriebe $a b$ fünf Beschäftigte. Die Größenabgrenzung wurde gewählt, um eine $\mathrm{Ge}-$ genüberstellung mit den Zahlen zur Verbreitung eines Betriebsrats zu ermöglichen. Zudem spielen solche Einrichtungen in den Kleinstbetrieben ohnehin so gut wie keine Rolle. Das schon angesprochene Nebeneinander von Betriebsrat und "hausgemachter" Mitarbeitervertretung gibt es allerdings nur bei einem Anteil von weniger als einem Prozent der Betriebe (ohne Tabelle). Wie nicht anders $\mathrm{zu}$ erwarten, kommt dies aber in größeren Betrieben deutlich häufiger vor (in Betrieben über 500 Beschäftigte bei ca. $6 \%$ ).

Die in den alten Bundesländern wesentlich stärkere Verbreitung betriebsspezifischer Mitarbeitervertretungen drückt sich auch in den auf die Beschäftigten bezogen Zahlen aus. Zurzeit arbeiten in Westdeutschland $11 \%$ der Beschäftigten in Betrieben mit einer solchen Einrichtung; in Ostdeutschland sind es $7 \%$.

Anders als beim Betriebsrat gibt es hier keine eindeutige größenspezifische Verteilung. In der Branche mit der stärksten Ver- breitung, den sonstigen Dienstleistungen, kommen immerhin rund $17 \%$ der Beschäftigten in den Genuss einer solchen Einrichtung, in den übrigen Branchen bewegen sich die Anteilswerte um die 10 Prozentmarke (Tabelle 5). Diese Zahlen können natürlich keine Auskunft über die Ausgestaltung der betreffenden Gremien oder gar deren betriebspolitische Rolle geben. Dazu sei auf andere Erhebungen verwiesen. ${ }^{2}$

\section{DAS ZUSAMMENSPIEL VON BETRIEBLICHER UND ÜBERBETRIEB- LICHER INTERESSENVERTRETUNG}

Im abschließenden gemeinsamen Blick auf die betriebliche und sektorale Ebene der (gesetzlich legitimierten) Interessenvertretung soll vor allem auch ein Eindruck von den Verschiebungen im dualen System gegeben werden. Damit gilt im Folgenden das Augenmerk vor allem der Entwicklung der sogenannten betrieblichen Vertretungslücken. Hierbei geht es um Betriebe, die zwar tarifgebunden sind, aber über keinen Betriebsrat verfügen, und den sogenannten weißen Flecken in der Tarif- und Mitbestimmungslandschaft, wo es keinerlei gesetzlich legitimierte Interessenvertretung gibt. Die präsentierten Zahlen beziehen sich ebenfalls auf die Betriebe der Privatwirtschaft $\mathrm{ab}$ fünf Beschäftigte. Eine Berücksichtigung der Kleinstbetriebe, die ja nicht zur Wahl eines Betriebsrats berechtigt sind, würde bei der gemeinsamen Betrachtung des Deckungsgrades von betrieblicher Mitbestimmung und Tarifbindung ein systematisch verzerrtes Bild liefern.

Wie sich auch in den aktuellen Zahlen bestätigt, ist nur eine Minderheit der in der Privatwirtschaft ( $a b$ fünf Beschäftigte) tätigen Arbeitnehmer in Betrieben eingestellt, die zur Kernzone des dualen Systems der
Interessenvertretung zu zählen sind. Diese Zone umfasst ein Drittel der Beschäftigten in Westdeutschland und nur ein Fünftel in Ostdeutschland (Tabelle 6). Bemerkenswert ist die mit $10 \%$ relativ große Bedeutung von Betrieben mit Haustarifen und einem Betriebsrat in den neuen Bundesländern. Von einer betrieblichen Vertretungslücke (Betrieb ist an einen Branchentarif gebunden, verfügt aber über keinen Betriebsrat) ist gut jeder fünfte Beschäftigte in Westdeutschland (22\%) aber nur etwa jeder sechste in Ostdeutschland (16\%) betroffen. Diese vermeintlich günstigere Situation in den neuen Bundesländern ist allerdings nur der dort geringeren Reichweite der Tarifbindung geschuldet, die sich in deutlicher ausgeprägten „weißen Flecken der Tarif- und Mitbestimmungslandschaft" äußert. Während in Westdeutschland "nur" $31 \%$ der Beschäftigten ohne Branchentarif und ohne Betriebsrat auskommen müssen, sind dies in Ostdeutschland $42 \%$.

Eine Betrachtung dieser Zahlen über die Jahre hinweg zeigt einige bemerkenswerte Entwicklungen. Die Kernzone des dualen Systems ist seit 1996 in beiden Landesteilen deutlich geschrumpft. Zuletzt gab es aber eine Phase der Konsolidierung, wenn gleich in Ostdeutschland auf deutlich niedrigerem Niveau als im Westen.

Ebenfalls abgenommen hat in den letzten zwölf Jahren der Umfang der betrieblichen Vertretungslücken, wenngleich sich

2 Vgl. Hauser-Ditz, A./Hertwig, M./Pries, L. (2006): Betriebsräte und andere Vertretungsorgane im Vergleich - Strukturen, Arbeitsweisen und Beteiligungsmöglichkeiten, in WSI-Mitteilungen 9 , S. 500-506; Schlömer, N./Kay, R./Rudolph, W./ Wassermann, W. (2008): Arbeitnehmerbeteiligung in mittelständischen Unternehmen, in: WSIMitteilungen 5, S. 254-260. 
auch hier in den letzten Jahren nicht mehr viel getan hat. Dieser Rückgang muss natürlich im Zusammenhang mit den deutlich angewachsenen ,weißen Flecken in der Tarif- und Mitbestimmungslandschaft" gesehen werden, wodurch immer weitere Teile der Betriebslandschaft ohne jegliche Form der gesetzlich legitimierten Interessenvertretung bleiben. Ein weiteres
Segment, das im Beobachtungszeitraum Zuwächse zu verzeichnen hat, umfasst Betriebe, die nicht (mehr) an einen Branchentarif gebunden sind, aber über einen Betriebsrat verfügen. All diese Trends sind zum weitaus größten Teil dem Rückgang der Branchentarifbindung geschuldet, bei parallel weitgehend stabiler Reichweite der betrieblichen Mitbestimmung. Insgesamt lässt sich aber sagen, dass sich die starken Veränderungen bzw. Verluste bis Anfang der 2000er Jahre ereignet haben. In den letzten Jahren kam es dagegen vor allem in Ostdeutschland zu einer Konsolidierung. Die weitere Entwicklung muss zeigen, ob es sich dabei vielleicht nur um eine Verschnaufpause handelt. 